\title{
Suspected transmission of tuberculosis in a maternity ward from a smear-positive nurse: preliminary results of clinical evaluations and testing of neonates potentially exposed, Rome, Italy, 1 January to 28 July 2011
}

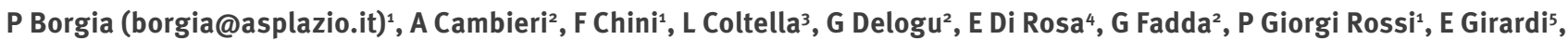

D Goletti ${ }^{5}$, G Guasticchi ${ }^{1}$, A Morrone ${ }^{6}$, P Pezzotti ${ }^{1}$, C Romagnoli ${ }^{2}$, M T Sacerdote ${ }^{4}$, C Russo ${ }^{3}$, A Villani ${ }^{3}$, L Zarelli ${ }^{5}$

1. Agency for Public Health, Lazio Region, Rome, Italy

2. Università Cattolica del Sacro Cuore, Rome, Italy

3. Bambino Gesù Children's Hospital, Rome, Italy

4. Azienda Sanitaria Locale Roma E, Rome, Italy

5. L. Spallanzani National Institute for Infectious Diseases (INMI), Rome, Italy

6. Azienda Ospedaliera S. Camillo-Forlanini, Rome, Italy

Citation style for this article:

Borgia P, Cambieri A, Chini F, Coltella L, Delogu G, Di Rosa E, Fadda G, Giorgi Rossi P, Girardi E, Goletti D, Guasticchi G, Morrone A, Pezzotti P, Romagnoli C,

Sacerdote MT, Russo C, Villani A, Zarelli L. Suspected transmission of tuberculosis in a maternity ward from a smear-positive nurse: preliminary results of clinical

evaluations and testing of neonates potentially exposed, Rome, Italy, 1 January to 28 July 2011.

Euro Surveill. 2011;16(40):pii=19984. Available online: http://www.eurosurveillance.org/ViewArticle.aspx?Articleld=19984

Article published on 6 October 2011

We report preventive measures adopted after tuberculosis (TB) transmission from a nurse to a newborn assessed in late July 2011. All exposed neonates born between January and July 2011 were clinically evaluated and tested by QuantiFERON TB gold in-tube; newborns testing positive were referred for prophylaxis. Of 1,340 newborns, 118 (9\%) tested positive and no other active cases of TB were found. Active surveillance for TB will be continued over the next three years for all those exposed.

\section{Case descriptions}

Case one

On 18 July 2011, a case of pulmonary and extra-pulmonary (splenic) tuberculosis (TB) was diagnosed in a fourmonth old infant at the children's hospital 'Bambino Gesù' in Rome, Italy. The diagnosis was confirmed by microscopic and sputum culture. According to international guidelines for TB prevention [1], all household members (parents and siblings, and two close contacts who took care of the baby) were screened for TB. No active TB cases were found and only the mother had a positive tuberculin skin test (TST) but was QuantiFERON TB gold in-tube (QFT-IT) TB-negative, most likely due to Bacillus Calmette-Guérin (BCG)-vaccination as a child. Although they tested negative, the two siblings, both below five years of age, were referred for prophylaxis.

\section{Case two}

On 26 July, pulmonary TB was clinically diagnosed in a patient at 'Gemelli' hospital, Rome and hereafter microbiologically confirmed by sputum culture as Mycobacterium tuberculosis, sensitive to first-line drugs, at the National Institute for Infectious Diseases
L. Spallanzani, Rome. The patient, a nurse by profession, reported onset of night cough since the beginning of April 2011 and a mild asthenia that was attributed to the daily work and diet, as well as loss of $17 \mathrm{~kg}$ body weight (corresponding to almost $20 \%$ of the total weight). The loss of weight was interpreted as intentional since she had been on a low-calorie diet since February 2011. In June 2011, a sinusitis had been diagnosed and beclometasone aerosol prescribed.

As soon as the nurse was suspected as a case of TB, investigation of household members found her husband and son TST-positive. Furthermore, the husband had a history of exudative pleurisy in 2004. Pleural fluid and sputa had resulted negative for $M$. tuberculosis isolation by culture at that time; the husband had nevertheless undergone a full course of TB therapy, consisting of two months of quadruple therapy with isoniazid, rifampicin, ethambutol and pyrazinamide, followed by four month of double therapy with isoniazid and rifampicin with a supplementation of vitamin B over the whole six months. Subsequently, he had successfully recovered from lung lesions, as assessed by radiology, and in terms of clinical symptoms. Therefore he had been diagnosed with pleural TB based on clinical grounds. At that time, the nurse was not tested by TST since her husband was deemed not contagious.

Because the nurse had been working in the maternity ward, at the same university hospital where the child (case one) was born in March 2011, an epidemiological link between the two cases was suspected. DNA fingerprinting of the isolated and cultured samples of $M$. tuberculosis in the two cases confirmed the link. 


\section{Epidemiological background}

Italy is a low TB prevalence country according to the World Health Organization (WHO) definition [2,3]. The annual incidence in Lazio (the region including Rome, 5.6 million inhabitants), taking into account underreporting, has been estimated to be around 11/100,000 [4]. Only 30 cases in children under one year of age have been reported annually to the local health authorities and regional public health agency in the last five years $(12 / 100,000)[5]$.

Very few nosocomial outbreaks involving newborns have been described to date [6-14]. Here we report a case of TB that occurred in a four-month old infant potentially linked to a nurse diagnosed with TB who worked in the maternity ward when the child was born.

\section{Investigation of potentially exposed newborns}

Immediately after diagnosis of pulmonary TB in the nurse working in the maternity ward, a task force, including public health authorities, hospital infection control authority, and experts from the paediatric and infectious disease reference centres, defined the protocol described below for epidemiological investigation and preventive treatment.

The exposed group was defined as all the newborns $(n=1,738)$ who were cared for in the maternity ward from three months before symptoms onset in the nurse to two days after the nurse's last working day (i.e. from 1 January to 28 July, 2011). Parents were contacted by phone and by mail, and counselled about the potential risk of infection and invited for a clinical visit and a screening test for the child in three paediatric outpatient clinics located in three different hospitals in Rome.

The task force decided to use QFT-IT (Cellestis Limited, Carnegie, Victoria, Australia) to screen newborns for several reasons: first, previous outbreak investigations of maternal wards suggested a lower sensitivity to latent TB infection of TST in neonates than in adolescents or adults $[6-8,15]$; second, it has been suggested to be more accurate than TST in low- and middleincome countries on children [16]; and, third it does not require, as does the TST, a second visit to assess results, which may be problematic for such a large population. However, there are very few studies of QFT-IT accuracy in newborns $[16,17]$. In any case, all children were tested by QFT-IT from 18 August. Since birth and potential exposure to M. tuberculosis were concurrent, and exposure was limited to one to two days during the hospitalisation in most cases, in the neonates below 12 weeks of age tested at the screening in August, a second QFT-IT and clinical evaluation were planned at the completion of three months of age. This is in agreement with guidelines that indicate an evaluation of contacts after the completion of three months from exposure.
The cut-off value for a positive test was 0.35 International Units(IU)/mL of interferon (IFN)-gamma in the sample after stimulation with the specific antigens, as suggested by the manufacturer. The task force decided to refer only those children positive to QFT-IT for isoniazid prophylaxis, since the exposed cohort had a priori a quite low probability of infection. Protocol dictated that children with a positive QFT-IT were referred for a chest X-ray and an additional clinical visit. An educational programme for the paediatricians of the exposed children, covering the clinical aspects of TB in that age group, was planned.

Tests whose results are reported here, were all performed from 18 August to 10 September, 2011. The table shows preliminary results of 1,340 tests for neonates whose parent or guardian consented to testing and examination. More potentially exposed neonates were tested after 10 September but the results are not yet known. Up to 10 September, a total of 118 (9\%) positive QFT-ITs were obtained and only three (0.2\%) indeterminate (low Mitogen Response, i.e. $0.5 \mathrm{IU} / \mathrm{mL}$ ). The three children were retested after a month, after which they were responsive to the mitogen, and negative to QFT-IT. There was no clear trend from exposure (birth) month. Due to the immaturity of immune system, it was surprising to find that neonates scored QFT-IT positive under five weeks of age $(11 \%, 9 / 79)$.

It is of note that, although not specified in the protocol, most QFT-IT positive infants subsequently were tested also with the TST, to check if the TST would confirm the positive QFT-IT. The infant diagnosed with active TB was both QFT-IT- and TST-positive, while the children

\section{TABLE}

Results of QuantiFERON TB gold by month of birth in the cohort of children potentially exposed to a nurse with active tuberculosis, Rome, Italy, January-July 2011 $(\mathrm{n}=1,340)$

\begin{tabular}{|l|c|c|}
\hline \multirow{2}{*}{ Month of birth in 2011 } & \multicolumn{2}{|c|}{ QuantiFERON TB gold } \\
\cline { 2 - 3 } & Total tested & Positive \\
\cline { 2 - 3 } & $\mathrm{N}^{\mathrm{a}}$ & $\mathrm{N}(\%)$ \\
\hline January & 122 & $15(12)$ \\
\hline February & 170 & $20(12)$ \\
\hline March & 209 & $21(10)^{\mathrm{b}}$ \\
\hline April & 214 & $13(6)$ \\
\hline May & 224 & $15(7)$ \\
\hline June & 223 & $15(7)$ \\
\hline July & 174 & $19(11)$ \\
\hline Unknown & 4 & $0(0)$ \\
\hline Total & $\mathbf{1 , 3 4 0}$ & $\mathbf{1 1 8 ( 9 )}$ \\
\hline
\end{tabular}

a Three childrens' samples with low Mitogen Response (i.e. $<0.5$ $\mathrm{IU} / \mathrm{mL}$ ) were considered indeterminate; after a month these children were retested after which they were mitogen-responsive and QuantiFERON in-tube negative. For 15 cases, negative to gamma-interferon, Mitogen was not performed and for one case the result was missing.

b Table does not include the only confirmed case of active tuberculosis that occurred in an infant born in March. 
without active TB, who had a QFT-IT positive result, were TST negative. To date, no other person with active TB has been identified.

Unfortunately there are no estimates of QFT-IT sensitivity and specificity in newborns [16-20]. Therefore, to estimate the proportion of true and false positives, QFT-IT test results from a sample of unexposed children of the same age are needed, but ethical and practical issues make this problematic. On the other hand, the sensitivity of immune-based tests is known to be low, in particular in the first weeks after infection and birth. Furthermore, it is possible that some infected children test false negative to QFT-IT. For these cases an active surveillance of symptoms by parents and paediatricians has been planned.

In conclusion, in this investigation 118 of 1,340 (9\%) newborns who had been exposed to a nurse with active TB were found to have a positive QFT-IT. The majority of the neonates with a positive QFT-IT were tested also by TST and found to be TST-negative. After two to nine months of follow-up, none of the QFT-IT-positive neonates progressed to active TB, indicating a low predictive value of this assay for progression to active TB in this population. The only case of active TB found in this cohort was positive, at the time of TB diagnosis, to both TST and QFT-IT. This cohort will be carefully followed for the next three years.

\section{References}

1. National Collaborating Centre for Chronic Conditions. Tuberculosis: Clinical Diagnosis and Management of Tuberculosis, and Measures for its Prevention and Control. London: Royal college of Physicians; 2006.

2. World Health Organization(WHO). Global tuberculosis controlsurveillance, planning, financing. WHO report 2007. Geneva: WHO; 2007. [Accessed 6/10/2011]. Available from http://www. who.int/tb/publications/global_report/2007/pdf/full.pdf

3. Moro M L, Malfait P, Salamina G, D’Amato S. Tuberculosis in Italy: available data and open questions. Epidemiol Prev. 1999;23(1): 27-36.

4. Farchi S, Mantovani J, Borgia P, Giorgi Rossi P. Tuberculosis incidence, hospitalisation prevalence and mortality in Lazio, Italy, 1997-2003. Int J Tuberc Lung Dis. 2008;12(2):193-8.

5. Giorgi Rossi P, Mantovani J, Ferroni E, Forcina A, Stanghellini E, Curtale F, et al. Incidence of bacterial meningitis (2001-2005) in Lazio, Italy: the results of a integrated surveillance system. BMC Infect Dis. 2009;9:13.

6. Nivin B, Nicholas P, Gayer M, Frieden TR, Fujiwara PI. A continuing outbreak of multidrug-resistant tuberculosis, with transmission in a hospital nursery. Clin Infect Dis. 1998;26(2):303-7.

7. Sen M, Gregson D, Lewis J. Neonatal exposure to active pulmonary tuberculosis in a health care professional. CMAJ. 2005;172(11):1453-56.

8. Richeldi L, Ewer K, Losi M, Bergamini BM, Roversi P, Deeks J, et al. T Cell-Based Tracking of multidrug resistant tuberculosis infection after brief Exposure. Am J Respir Crit Care Med. 2004;170(3):288-95.

9. Lee LH, LeVea CM, Graman PS. Congenital tuberculosis in a neonatal intensive care unit: case report, epidemiological investigation, management of exposures. Clin Infect Dis. 1998;27(3):474-7.

10. Mihăilescu P, Calciu B, Ionescu J. [Epidemic of tuberculosis in a maternity hospital triggered by congenital tuberculosis in a newborn infant]. Rev Ig Bacteriol Virusol Parazitol Epidemiol Pneumoftiziol Pneumoftiziol. 1979;28(2):85-93. [Article in Romanian]
11. Burk JR, Bahar D, Wolf FS, Green, J, Bailey WC. Nursery exposure of 528 newborns to a nurse with pulmonary tuberculosis. South Med J. 1978;71(1):7-10.

12. Light IJ, Saidleman M, Sutherland JM. Management of newborns after nursery exposure to tuberculosis. Am Rev Respir Dis. 1974;109(4):415-9.

13. Steiner P, Rao M, Victoria MS, Rudolph N, Buynoski G. Miliary tuberculosis in two infants after nursery exposure: epidemiologic, clinical, and laboratory findings. Am Rev Respir Dis. 1976;113(3):267-71.

14. Laartz BW, Narvarte HJ,Holt D, Larkin JA, Pomputius WF 3rd. Congenital tuberculosis and management of exposures in a neonatal intensive care unit. Infect Control Hosp Epidemiol. 2002;23(10):573-9.

15. Richeldi L, Ewer K, Losi M, Bergamini BM, Millington K, Fabbri LM, et al. T-cell-based diagnosis of neonatal multidrug-resistant latent tuberculosis infection. Pediatrics. 2007;119(1):e1-5.

16. Mandalakas AM, Detjen AK, Hesseling AC, Benedetti A, Menzies D. Interferon-gamma release assays and childhood tuberculosis: systematic review and meta-analysis. Int J Tuberc Lung Dis. 2011; 15(8):1018-32.

17. Machingaidze S, Wiysonge CS, Gonzalez-Angulo Y, Hatherill M, Moyo S, Hanekom W, et al. The utility of an interferon gamma release assay for diagnosis of latent tuberculosis infection and disease in children: a systematic review and meta-analysis. Pediatr Infect Dis J. 2011;30(8):694-700.

18. Bianchi L, Galli L, Moriondo M, Veneruso G, Becciolini L, Azzari $C$, et al. Interferon-gamma release assay improves the diagnosis of tuberculosis in children. Pediatr Infect Dis J. 2009;28(6): 510-4.

19. Connell TG, Curtis N, Ranganathan SC, Buttery JP. Performance of a whole blood interferon gamma assay for detecting latent infection with Mycobacterium tuberculosis in children. Thorax. 2006;61(7):616-20.

20. Detjen AK, Keil T, Roll S, Hauer B, Mauch H, Wahn U, et al. Interferon-gamma release assays improve the diagnosis of tuberculosis and nontuberculous mycobacterial disease in children in a country with a low incidence of tuberculosis. Clin Infect Dis. 2007;45(3):32 